\title{
Analysis of Stress and Displacement of Roadway with Prestressed Bolting by 3D-FSM
}

\section{C.WANG}

Network and Technology Center, Shandong University of Science and Technology, Qingdao, China

C.L. LIU

College of Mining and Safety Engineering, Shandong University of Science and Technology, Qingdao, China

\begin{abstract}
The fictitious stress method (FSM) based on analytical integral of Kelvin's solutions to calculate displacements or stresses have been proposed by K.Kuriyama. The paper represents the procedure how to use this method to compute stresses and displacements of rocks underground, and a modeling method for tunnel and bolt drilling element. In additional, some roadway with one prestressed bolting is taken as example and plot the $S_{z z}$ component of stress compared with Flac3d result. The FSM which employs integral of Kelvin's solution as influence coefficients is a calculation method of high accuracy, and the computation time complexity is lower compared with either the finite element method or finite difference method.
\end{abstract}

KEYWORD: fictitious stress method; analytical integrals; triangular leaf elements; BEM

\section{INSTRUCTION}

The original rock stress around roadways is complexly composed by gravitational field, reconstruction stress field, temperature field and so on. The stability analysis of underground caverns and roadways often employs approximate numerical simulation methods. So a problem is raised that the error brought by approximate simulation will be great if the model is divided into big cells, and meanwhile it will take too much time if cell division is too small when model is large enough. A twodimensional plane FSM method based on integration of Kelvin's solution is proposed by SL.Crouch[1]. And K.Kuriyama and Liu Chenglun have given a new fictitious stress method in three-dimensional in their papers[2-3], which employs analysis integral influence coefficients instead of analog numerical integration method. The FSM can be effectively used to solve singularity problems about integration of Kelvin's solutions. It is depended on exact influence coefficients, and greatly reduces the time complexity. This paper proposes how to use this method to solve the problem of stress and displacement around roadway, including numerical simulation procedure, establishment of the model, and usage on bolt preload stress affect in the rock around roadway.

\section{BOUNDARY INTEGRALS EQUATIONS}

The fictitious stress method is a kind of indirect boundary element method (IBEM), which has been usually used for prediction of the elastic behaviour of rocks. In these boundary element methods' procedures, numerical integrations are taken. This paper tells about a fictitious stress method procedure in which analytical integrals are developed to solve three-dimensional elastic problems.

Suppose a group of fictitious resultant tractions applied to the surface $\Gamma$ of an isotropic elastic solid illustrated in Figure 1.

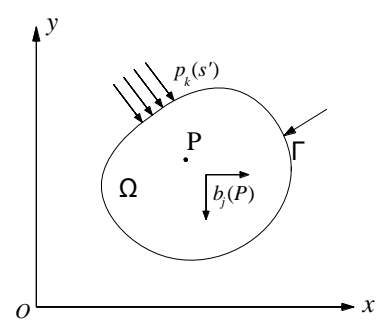

Figure1. Force sketch map of elastic body

The stresses and displacements induced by the tractions can be given:

$$
\begin{aligned}
& u_{l}(P)=\int_{\Gamma} \sum_{k=1}^{3} p_{k}(Q) u_{l k}^{*}(P, Q) d \Gamma(Q) \\
& +\int_{\Omega} \sum_{k=1}^{3} b_{k}(Q) u_{l k}^{*}(P, Q) d \Omega(Q)+C_{l} \quad(l=1 \sim 3) \\
& \sigma_{l m}(P)=\int_{\Gamma} \sum_{k=1}^{3} p_{k}(Q) \sigma_{l m k}^{*}(P, Q) d \Gamma(Q) \\
& +\int_{\Omega} \sum_{k=1}^{3} b_{k}(Q) \sigma_{l m k}^{*}(P, Q) d \Omega(Q), \quad(l, m=1 \sim 3)
\end{aligned}
$$


where $U_{l}(P)$ and $\sigma_{l m}(P)$ are the displacement and stress components of the plane, and $p k, b k$ are the components of the resultant tractions and body force, and $U^{*}{ }_{l k}(P, Q)$ are the Kelvin's Solutions.Omitting the body force and the displacement of the rigid, the stress and the displacement of point $\mathrm{P}$ can be calculated, viz.

$$
u_{l}(P)=\int_{\Gamma} \sum_{k=1}^{3} p_{k}(Q) u_{l k}^{*}(P, Q) d \Gamma(Q) \quad(l=1 \sim 3)
$$

\section{SOLUTION PROCEDURE OF 3D-FSM}

Discrete approximations are imaged to continuous distribution of stress that exists over triangular leaf elements which divided from the solid's boundary. The unknown variables $p_{x}^{i}, p_{y}^{i}, p_{z}^{i}$ are the components of the fictitious stress at the ith element. Then set up a new local co-ordinate system for the ith element, in which the points $P_{1}, P_{2}, P_{3}$ are arranged anticlockwise. $P_{1}$ is taken as origin of the coordinate. The direction of the vector $\vec{A}=\overrightarrow{\mathrm{P}_{1} \mathrm{P}_{2}}$ is defined as $\mathrm{X}$-axis, and $\overrightarrow{\boldsymbol{B}}=\overrightarrow{\mathbf{P}_{1} \mathbf{P}_{3}}$. The discrete equations are introduces as following Equations (4) and (5):

$$
\begin{aligned}
& {\left[\begin{array}{l}
u_{x}^{j} \\
u_{y}^{j} \\
u_{z}^{j}
\end{array}\right]=\sum_{i=1}^{n}\left[R_{j i}\right] \cdot\left[Q_{j i}\right] \cdot\left[\begin{array}{c}
p_{x}^{i} \\
p_{y}^{i} \\
p_{z}^{i}
\end{array}\right]} \\
& {\left[\begin{array}{c}
\sigma_{x}^{j} \\
\sigma_{y}^{j} \\
\sigma_{z}^{j} \\
\tau_{x y}^{j} \\
\tau_{y z}^{j} \\
\tau_{z x}^{j}
\end{array}\right]=\sum_{i=1}^{n}\left[T_{j i}\right] \cdot\left[S_{j i}\right] \cdot\left[\begin{array}{l}
p_{x}^{i} \\
p_{y}^{i} \\
p_{z}^{i}
\end{array}\right]}
\end{aligned}
$$

where $\left[R_{j i}\right],\left[T_{j i}\right]$ are the transformation matrixes about the displacement and stress from coordinate system $i$ to coordinate system $j$, and $\left[Q_{j i}\right],\left[S_{j i}\right]$ are the influence coefficient matrixes about the displacement and stress of the center of gravity at the $j$ th element in coordinate system $i$. The influence coefficient matrixes of 3D-FSM caused by triangular element $i$ can be written directly as Equations (6) and (7), and parameters $f_{1} \sim f_{17}$ which are derived through the integration of the triangular element base on Kelvin's solutions have been defined in literature (3).

$$
\left[Q_{i}\right]=\frac{1}{16 \pi G(1-v)}\left[\begin{array}{ccc}
f_{5}+(3-4 v) f_{1} & f_{7} & z f_{3} \\
f_{7} & f_{6}+(3-4 v) f_{1} & z f_{4} \\
z f_{3} & z f_{4} & z f_{2}+(3-4 v) f_{1}
\end{array}\right]
$$

$$
\begin{aligned}
& {\left[S_{i}\right]=\frac{1}{8 \pi G(1-v)}} \\
& {\left[\begin{array}{ccc}
-\left[(1-2 v) f_{3}\right]+3 f_{14} & (1-2 v) f_{4}-3 f_{17} & (1-2 v) f_{2}-3 f_{11} \\
\left.(1-2 v) f_{3}\right]-3 f_{16} & -\left[(1-2 v) f_{4}+3 f_{15}\right] & (1-2 v) f_{2}-3 f_{12} \\
\left.(1-2 v) f_{3}\right]-3 f_{9} & (1-2 v) f_{4}-3 f_{10} & -\left[(1-2 v) f_{2}+3 f_{8}\right. \\
-\left[(1-2 v) f_{4}+3 f_{17}\right] & -\left[(1-2 v) f_{3}\right]+3 f_{16} & -3 z f_{13} \\
-3 z f_{13} & -\left[(1-2 v) f_{2}+3 f_{12}\right] & -\left[(1-2 v) f_{4}+3 f_{10}\right] \\
-\left[(1-2 v) f_{2}+3 f_{11}\right] & -3 z f_{13} & -\left[(1-2 v) f_{3}+3 f_{9}\right]
\end{array}\right]}
\end{aligned}
$$

The procedure how to calculate components of stress can be described as:

Step.1 Let the solid boundary be divided into $n$ elements in triangular leaf shape connecting with each other. Displacement and stress induced at the center of gravity of the $j$ th element can be given, but they are represented by the local coordinates of the ith element. Assume the components of stress evenly distributed as $p_{x}^{i}, p_{y}^{i}, p_{z}^{i}$. Transform the forces induced by elements $1 \sim n$ to $j$ th local coordinate system and sum them, we can get the right expressions of Equations (4) and (5).

Step. 2 The stress in rocks will be changed once tunnel is excavated. The redistributed stress $([\sigma])$ equals the sum of initial stress $\left[\sigma_{0}\right]$ and induced stress $\left[\sigma_{1}\right]$, viz. $[\sigma]=\left[\sigma_{0}\right]+\left[\sigma_{1}\right]$. There are $3 \times n$ unknown variables including $p_{x}^{i}, p_{y,}^{i}, p_{z}^{i}(i=1 \sim n)$. For the solutions, we set up $3 \times n$ simultaneous equations as the following:

$$
\left[\begin{array}{c}
p_{0}^{j}-\sigma_{z 0}^{j} \\
-\tau_{y z 0}^{j} \\
-\tau_{z x 0}^{j}
\end{array}\right]=\sum_{i=1}^{n}\left[T_{j i}\right] \cdot\left[S_{j i}\right] \cdot\left[\begin{array}{c}
p_{x}^{i} \\
p_{y}^{i} \\
p_{z}^{i}
\end{array}\right], \quad(j=1 \sim n)
$$

where $p_{x}^{i}, p_{y,}^{i} p_{z}^{i}$ are the stress components of the plane on the ith element, which are induced by far field stress and $P^{j}{ }_{0}$ is the internal fluid pressure applied to ith element on the boundary. The stress and displacement induced by excavation on the boundary can be calculated by substituting the stress discontinuities obtained from Equation (8).

Step.3 With the value of $p_{x}^{i}, p_{y}^{i}, p_{z}^{i}(i=1 \sim n)$, we can calculate all six components of stress of an arbitrary point with the Equation (9):

$$
\left[\sigma^{p}\right]=\sum_{i=1}^{n}[T][S]\left[\begin{array}{c}
p_{x}^{i} \\
p_{y}^{i} \\
p_{z}^{i}
\end{array}\right]+\left[\sigma_{0}^{p}\right]
$$

Where $[T]$ is the coordinate transformation matrixes from coordinate system $i$ to global coordinate system. $[S]$ is the relevant influence coefficient matrix.

\section{MODELLING}

For example, we need to set up an arched linear monomer tunnel with cross-section composed by semi-circular above and rectangular portion below. The coordinate system is set up illustrated in 
Figure2. From the origin, a series of points can be sequentially sampled and sorted in the counterclockwise direction. For the semi-circular portion of the partition, someone suggested to division as shown in Figure 2(b). But by the method the step distance of semi-circular portion is too long and will result in serious error.

To reduce the affection of the above error, we can divide in an unique step distance same to the bottom: Supported that roadway's width is $W$, the radius of a circle $R=\frac{W}{2}$; if the bottom is divided into $C$ segments, the step size will be $\frac{W}{C}$. By the formula of arch length $\frac{W}{C}=r * \theta$, the rotation angle of each step is obtained $\theta=2 / C$. This approach ensures the edge length of semi-circle consistent with the bottom edge, but there may be a new problem as shown in Figure 2(c) that the last two points may be too close to cause narrow triangle (point 14 and point 14 ' in close proximity). If the semi-circle is divided into $x$ units, according to the semi-circular with the same step size to the bottom, the equation can be set up: $S_{x}=\frac{\pi R}{x}=\frac{W}{C}=\frac{2 R}{C}$. It is derived that $x=\frac{\pi C}{2}$, and finally obtain the angular step $\theta=\frac{\pi}{\left[\frac{\pi C}{2}\right]}$ ( $[x]$ : rounding of $\mathrm{x}$ ), the cross section shown in Figure 2(d).
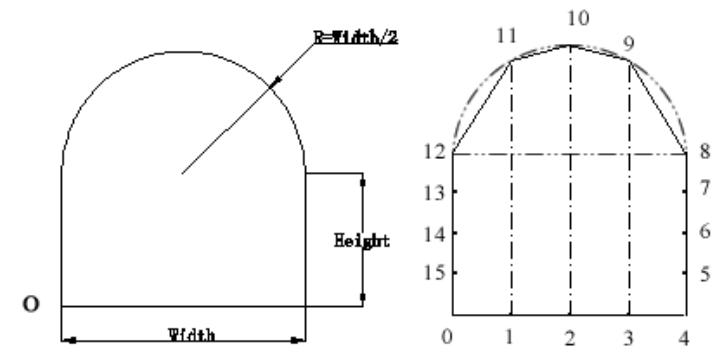

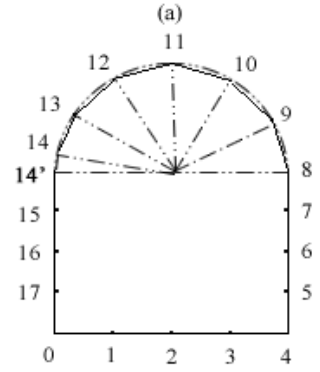

(c)

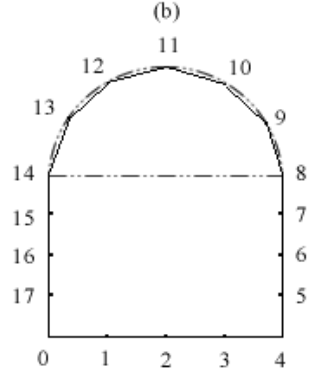

(d)
Figure 2. Several kinds of arrangements of base points

The set of points above is called the initial points set $A_{0}=\left\{\left(x_{i}, y_{i}, 0\right), i=0 . . m\right\}$. Where the upper bound value is $\mathrm{m}$ in the initial set of points, as shown in Figure $2(\mathrm{~d}), m=17$. Along the depth direction of tunnel, defined as $\mathrm{z}$ axis, a series of point sets $A_{1}, A_{2}, \ldots A_{k}, \ldots A_{n} \quad$ can be added, $A_{k}=\left\{\left(x_{i}, y_{i}, k * S_{z}\right), i=0 . . m\right\}$.

Finally, the set of points $A_{k}$ and $A_{k+1}$ are connected with the adjacent dots to form a triangle mesh. A separate division of triangulation is taken to the set of points $A_{0}$ and $A_{m}$ at both ends of the roadway.

so that all points are weaved as a closed entity as shown in Figure 3 (implemented in OpenGL).

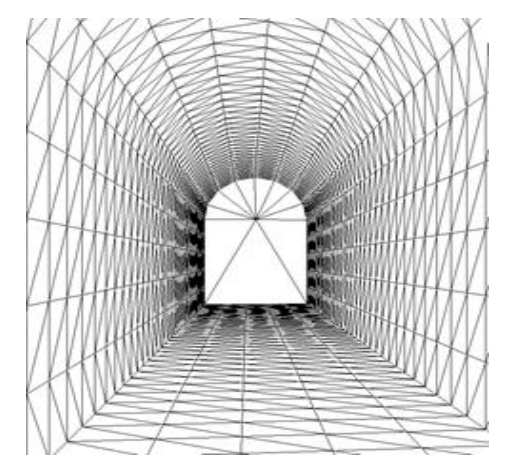

Figure 3. The Internal profile of tunnel

By the above method to draw two roadway entities intersect in the world coordinate system, in fact the Boolean operations of two entities, including collision detection, finding intersecting line, triangulation based on intersecting line and the choice of the triangular face satisfied to Boolean operations and other issues. Suppose roadway A and roadway B intersect to each other. To detect which triangular facets intersected with others, Several approaches can be employed mainly include spherical bounding box, axial bounding box (AABB), direction Bounding Box (OBB), discrete direction polyhedron (k-DOP) and Time-box and other methods[5-7]. The spherical and axial rectangular bounding box method are relatively simple applications, but not enough accurate. OBB and k-DOP methods are the more popular method in recent years, but more complicated, Zhang Shaoli[8] proposed an algorithm for improvement judgment on the part of the optimized trade-offs of intersection. After the bounding box collision detection, we need to detailedly calculate the intersection of triangular facets[9]. It is the appearance and inner profile graphs that shown in Figure 4 and Figure 5. Relative to the entire roadway, the diameter of bolt drilling hole is negligible, so we can use hole line element to simulate it.

\section{EXAMPLE}

As a case study of modeling for a roadway in some mine lot, the main tunnel is $100 \mathrm{~m}$ long and $5 \mathrm{~m}$ wide. The side-wall is $3 m$ high, and the diameter of 
semicircular arched roof is $5 \mathrm{~m}$. At the middle point of the roadway a bolt is set up at the center of roof. Let the FSM element in which the bolt lies be a tray unit with preload $f=1.5 \times 10^{5} \mathrm{~N}$. Without considering the elastic deformation of anchoring agent, by the above 3D-FSM, the component $S_{z z}$ of the rock around roadway's stress can be calculated as Figure6, compared with the plot by Flac3d shown in Figure7.

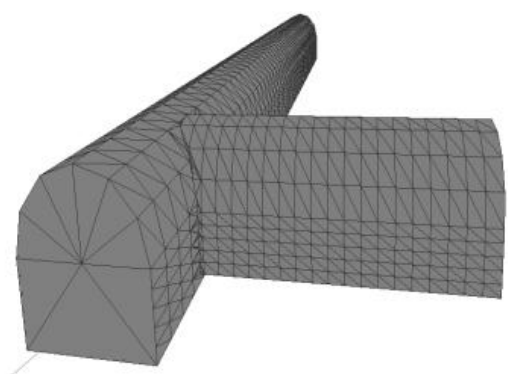

Figure 4. The appearance of tunnel intersection

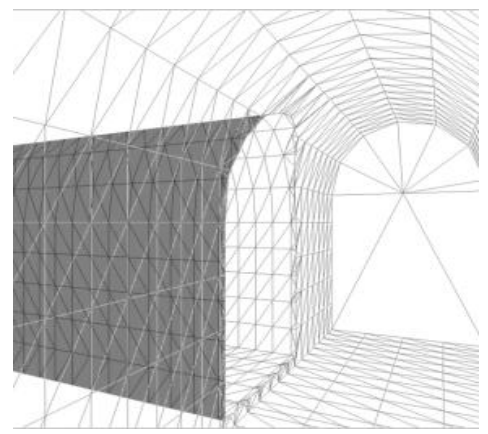

Figure 5. The inner profile of tunnel intersection

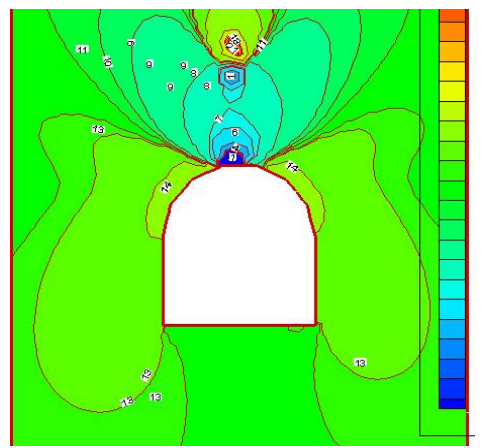

Figure 6. Contour plot of $S z z$ by FSM

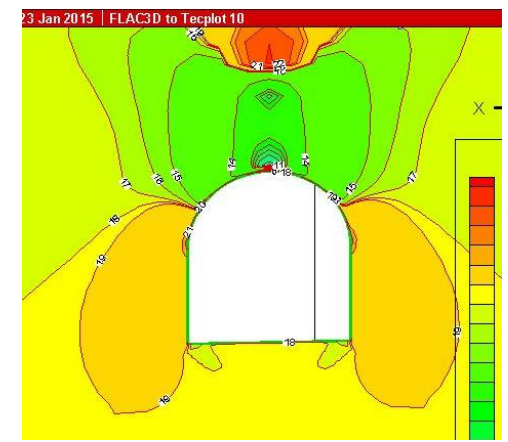

Figure 7. Contour plot of $S z z$ by Flac3d
By contrast with Flac3d chart, the result of the FSM numerical simulation method is correct and receivable. Because the 3D-FSM calculates stress or displacement based on the Kelvin equation, it can be seen that the curve is continuous and smooth, but the Flac3d graph in a partial region is not smooth, due to the computing procedure being based on hexahedral elements.

\section{CONCLUSION}

Kelvin's solution is the exact solution in the infinite domain about concentrated stress. The FSM which employs integrals of Kelvin's solution as influence coefficients is a calculation method of high accuracy. In addition, the computation time complexity is low compared with either the finite element method or finite difference method. It has specific advantages for solving the problems of hole and crack in an elastic body that by being combined with 3DDDM[4].

\section{REFERENCES}

[1] S.L.Crouch, A.M.Starfield, Boundary element methods in solid mechanics, London : Unwin Hyman, 1974,pp25-26

[2] K.Kuriyama, Y.Mizuta, H.Mozumi, T.Watanabe, Threedimensional Elastic Analysis by the Boundary Element Method with Analytical Integrations over Trianguluar Leaf Elements, journal of Int.J.Rock Mech.Min.Sci \& Geomech.Abstr.Vol.32,No.1,pp.77-83,1995

[3] Liu Chenglun, Y.Mizuta, K.Kuriyama, Anaytical Integrations for Three-dimensional Fictitious Stress Method Based on Kelvin Solution, Journal of the mining and materials processing institute of Japan.Vol.115,pp719724,1999

[4] K.Kuriyama, Y.Mizuta, Three-dimensional Elastic Analysis by the Displacement Discontinuity Method with Boundary Division into Triangular Leaf Elements, journal of Int.J.Rock Mech.Min.Sci \& Geomech. Abstr.Vol.30, No.2, pp.111-123,1993

[5] WANG Chun-lin, WANG Qing-lin, WANG Yu-kun, Establishment on Roaming Model of Mine 3D Roadway Based on OpenGL, Coal Science and Technology, 2009, 09:75-78.

[6] HUA Zhen, FAN Hui, LI Jin-jiang, Application of virtual reality technology in mine ventilation syste. Coal Science and Technology, 2004, 03: 44-48.

[7] YAO Jian-hai. Modeling Technology Study on the Threedimensional Laneway. Journal of Taiyuan University of Science and Technology, 2009, 01: 80-83.

[8] ZHANG Shao-li, WANG Yi-gang, CHEN Xiao-diao, Intersection method for triangular mesh model based on space division, Journal of Computer Applications, 2009, 10:2671-2673.

[9] Tomas M. A fast triangle-triangle intersection test. Journal of Graphics Tools, 1997, 2(2):25-30. 